\title{
Piezoelectricity and Pyroelectricity in a Copolymer of Vinylidene Fluoride and Tetrafluoroethylene
}

\author{
Jian Xun WEN* \\ Institute of Physical and Chemical Research, \\ Wako, Saitama 351-01, Japan
}

(Received July 19, 1984)

\begin{abstract}
The piezoelectric constants $d_{31}, d_{32}, d_{33}^{\mathrm{a}}$ were measured for the undrawn and uniaxially drawn copolymer of vinylidene fluoride $(80 \mathrm{~mol} \%)$ and tetrafluoroethylene $(20 \mathrm{~mol} \%)$ as a function of remanent polarization $P_{\mathrm{r}}$. The data of undrawn samples are consistent with the assumption that the piezoelectric activity mainly arises from macroscopic dimensional changes. Drawn samples show a strong in-plane anisotropy so that $d_{31}$ is much larger than $d_{32}$. Such an anisotropy is mostly attributable to anisotropic Poisson's ratio. The temperature dependence of the piezoelectric constants is discussed. The pyroelectric constant $p_{3}$ was measured as a function of $P_{\mathrm{r}}$. Using piezoelectric and thermal expansion data, the contribution from the secondary effect was calculated and found to be $1 / 4$ the total activity.

KEY WORDS Ferroelectricity / Piezoelectricity / Pyroelectricity /

Copolymer of Vinylidene Fluoride and Tetrafluoroethylene /
\end{abstract}

The strong piezoelectric and pyroelectric activities in uniaxially drawn and poled poly(vinylidene fluoride) (PVDF) have stimulated a great deal of interest in this polymer. $^{1,2}$ Several reviews on piezoelectricity and pyroelectricity have been published. ${ }^{3-6}$ The copolymer of vinylidene fluoride (VDF) with trifluoroethylene (TrFE) has been of special interest recently because it is ferroelectric toward paraelectric transition. We also investigated piezoelectric and pyroelectric responses of this copolymer. Since the homopolymer and copolymers of VDF are ferroelectric, their piezoelectric and pyroelectric activities are attributable to ferroelectric orientation of the crystalline dipoles. $^{7-10}$

The copolymer of vinylidene fluoride (VDF) with tetrafluoroethylene (TeFE) is known to have a crystalline phase analogous to the $\beta$ phase of PVDF of as-cast or melt-crystallized films. ${ }^{11}$ Baise and coworkers have shown that the copolymer exhibits higher pyroelectric activity than PVDF under the same poling conditions. ${ }^{12}$ Davis and Broadhurst measured the piezoelectric constant determined by the change in polarization with hydrostatic pressure and discussed the results on the basis of a. molecular theory. ${ }^{13}$ Collins and coworkers measured pyroelectric constants for the $73 / 27$ $\mathrm{VDF} / \mathrm{TeFE}$ copolymer and showed that the films are not uniformly poled across their thickness and that the response arises from two separate effects. ${ }^{14}$ Stefanou reported the film compliance, density, degree of crystallinity and thickness piezoelectricity for copolymers containing 61 to $96 \%$ VDF and concluded that the piezoelectric constant can be calculated from the molecular dipole theory where the polarization is derived using a cavity reaction field. ${ }^{15}$ Hicks and his coworkers have studied the ferroelectric properties of this copolymer. ${ }^{16}$ In comparison with PVDF, VDF/TeFE co-

\footnotetext{
* Permanent address: Shanghai Institute of Organic Chemistry, 345, Lingling Lu, Shanghai, China.
} 
polymer has been investigated to a lesser extent.

In this paper, we present further experimental results on piezoelectricity and pyroelectricity in undrawn and/or uniaxially drawn samples of a $\mathrm{VDF} / \mathrm{TeFE}$ copolymer. In contrast to conventional high temperature poling, we carried out room temperature poling using a slowly changing high electric field and prepared samples with known values of remanent polarization $P_{\mathrm{r}}$. We determined the transverse and longitudinal piezoelectric constants as a function of $P_{\mathrm{r}}$. The results allowed us to examine the contribution of the dimensional effect on piezoelectric activity. We also measured the temperature dependence of the piezoelectric constant as well as the dielectric and elastic constants of the copolymer to obtain further information. We also discuss the mechanisms of pyroelectric activity.

\section{EXPERIMENTAL}

\section{Sample Preparation}

The sample used was an 80/20 copolymer of VDF and TeFE supplied by Daikin Kogyo Co. Ltd. Undrawn film samples were obtained by casting from a methyl ethyl ketone solution and annealed at $120^{\circ} \mathrm{C}$ for $1 \mathrm{~h}$. The uniaxial drawing was performed at $45^{\circ} \mathrm{C}$ to 7 times their original length, followed by annealing at $100^{\circ} \mathrm{C}$ for $1 \mathrm{~h}$. Gold electrodes were evaporated in vacuo onto both sides of the samples.

\section{Measurements}

The poling of samples was undertaken at room temperature by applying a sinusoidal high electric field of $100^{-1} \mathrm{~Hz}$ up to 150 $\mathrm{MV} \mathrm{m}^{-1}$. By monitoring the induced charges, we obtained a typical hysteresis loop as shown in Figure 1. The remanent polarization $P_{\mathrm{r}}$ was ca. $60 \mathrm{mC} \mathrm{m}^{-2}$, which is much larger than the value reported by Hicks and coworkers. ${ }^{16}$ This may be because we applied a much higher field and induced better orientation of the dipoles. The coercive field $E_{\mathrm{c}}$ was found to be 50

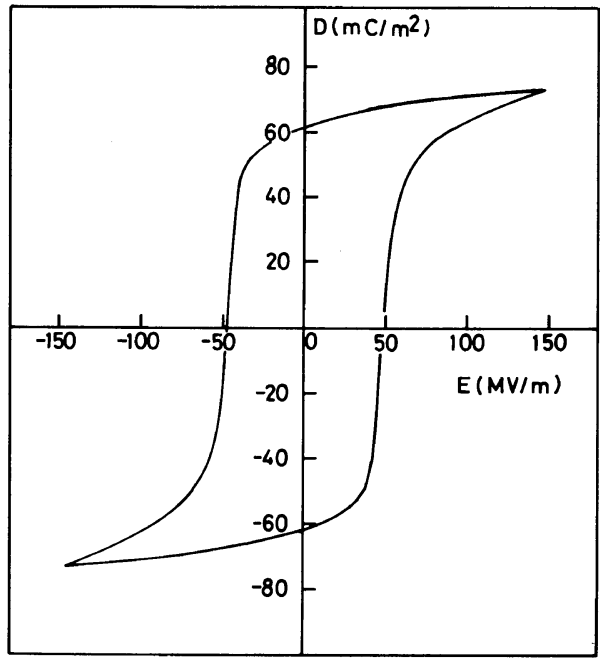

Figure 1. Hysteresis loop of an undrawn $80 / 20 \mathrm{VDF} /$ TeFE copolymer.

$\mathrm{MV} \mathrm{m}^{-1}$. By changing the maximum applied field, we prepared poled samples with various values of $P_{\mathrm{r}}$.

We studied two types of piezoelectric effects, the transverse effect and longitudinal effect. The transverse effects were observed at $10 \mathrm{~Hz}$ by the ratio of charge density generated on the electrode to the applied stress along the drawing direction and/or perpendicular to this direction in-plane of a rectangular sample. Thus, we obtained $d_{31}$ and $d_{32}$. At the same time, we measured Young's moduli $1 / s_{11}$ and $1 / s_{22}$ and the dielectric constant $\varepsilon_{3} / \varepsilon_{0}$. We used the Cartesian coordinate system where the 1 axis was taken along the draw direction and the 3 axis, along the thickness [poling direction]. The temperature dependence of the piezoelectric constant was measured from -150 to $100^{\circ} \mathrm{C}$. The longitudinal effect was measured by applying a quasi-static stress along the thickness direction to a sample sandwiched between a pair of glass plates. In this configuration, the in-plane deformation of the sample was restricted. Thus, we obtained $d_{33}^{\text {a }}$ rather than the true $d_{33}$. The pyroelectric constant $p_{3}$ was obtained by a conventional technique which measures the change in total 
charge on the electrodes due to an increase or decrease in the temperature of a mechanically free sample.

The most commonly used piezoelectric constants are defined by ${ }^{17}$

$$
\begin{aligned}
& d_{3 j}=\frac{1}{A}\left(\frac{\partial Q_{3}}{\partial X_{j}}\right)_{X_{i \neq j}=0} \\
& e_{3 j}=\frac{1}{A}\left(\frac{\partial Q_{3}}{\partial x_{j}}\right)_{x_{i \neq j}=0}
\end{aligned}
$$

where $X_{j}$ is the stress, $x_{j}$ the strain and $Q_{3}$, the total charge induced on the electrodes of area A. $d_{3 j}$ and $e_{3 j}$ are related to each other by ${ }^{18}$

$$
\begin{aligned}
& d_{3 j}=\sum_{i=1}^{3} e_{3 i} s_{i j} \\
& e_{3 j}=\sum_{i=1}^{3} d_{3 i} c_{i j}
\end{aligned}
$$

where $s_{i j}$ is the elastic compliance and $c_{i j}$, the elastic stiffness. We sometimes used the constants defined by ${ }^{19}$

$$
\begin{aligned}
& d_{3 j}^{\mathrm{a}}=\left(\frac{\partial P_{3}}{\partial X_{j}}\right)_{X_{i \neq j}=0} \\
& e_{3 j}^{\mathrm{a}}=\left(\frac{\partial P_{3}}{\partial x_{j}}\right)_{x_{i \neq j}=0}
\end{aligned}
$$

The superscript a denotes the apparent value of these constants because they differ from $d_{3 j}$ and $e_{3 j}$ in eq 1 and 2 in terms of mechanical boundary conditions. The details are described in a previous paper. ${ }^{19}$ Corresponding to eq 3 and 4 , we have

$$
\begin{gathered}
d_{3 j}^{\mathrm{a}}=e_{3 j} / c_{j j} \\
e^{\mathrm{a}}{ }_{3 j}=d_{3 j} / s_{j j}
\end{gathered}
$$

We measured three constants: $d_{31}$ and $d_{32}$ and $d_{33}^{\mathrm{a}}$. The other constants were calculated using eq $3,4,7$, and 8 .

\section{RESULTS}

The $\beta$-phase of PVDF is usually obtained by drawing melt crystallized films. Since the $\mathrm{VDF} / \mathrm{TeFE}$ copolymer was assumed to have a

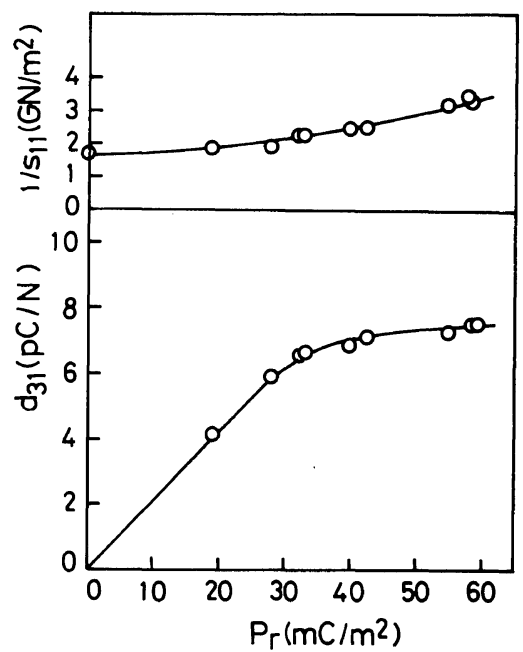

Figure 2. Dependence of the transverse piezoelectric constant $d_{31}$ and the Young's modulus $1 / s_{11}$ on the remanent polarization $P_{\mathrm{r}}$ in an undrawn VDF-TeFE copolymer.

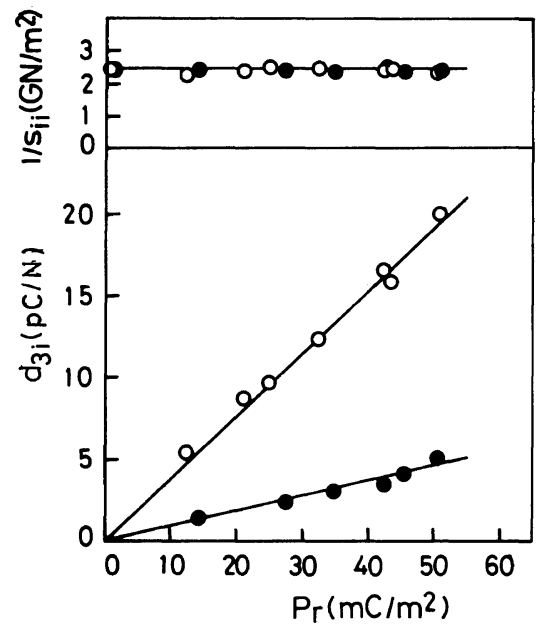

Figure 3. Dependence of $d_{3 i}$ and the Young's modulus $1 / s_{11}$ on $P_{\mathrm{r}}$ in uniaxially drawn $\mathrm{VDF} / \mathrm{TeFE}$ copolymer. $\bigcirc$, for $d_{31}$ and $1 / s_{11}$ respectively; $\bigcirc$, for $d_{32}$ and $1 / s_{22}$, respectively.

crystalline structure similar to the $\beta$-PVDF of as-cast or melt-crystallized films, we could observe a square $D-E$ hysteresis loop and piezoelectric and pyroelectric activities in undrawn samples. Figure 2 shows the dependence of the piezoelectric constant $d_{31}$ and Young's modulus $1 / s_{11}$ on the remanent po- 


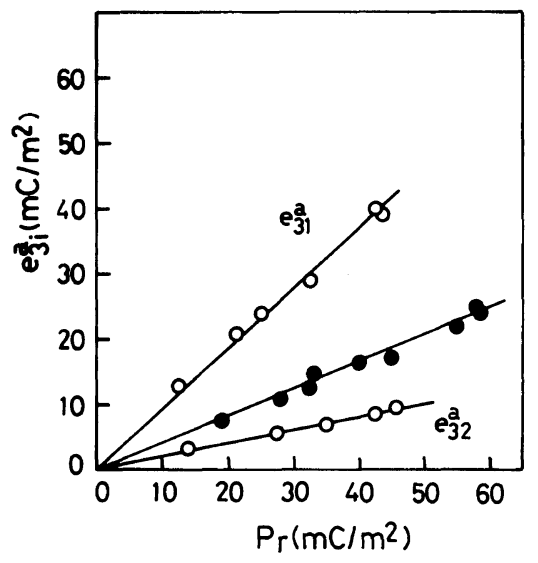

Figure 4. Dependence of $e_{3 i}^{\mathrm{a}}$ on $P_{\mathrm{r}}$ in undrawn and uniaxially drawn VDF/TeFE copolymers. for $e_{31}^{\mathrm{a}}$ of an undrawn copolymer.

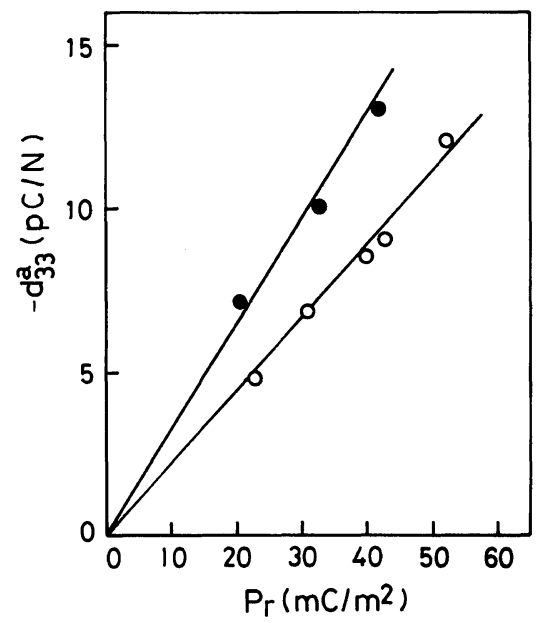

Figure 5. Dependence of $e_{33}^{\mathrm{a}}$ on $P_{\mathrm{r}}$ in undrawn and uniaxially drawn VDF/TeFE copolymers: $O$, for an undrawn copolymer; $\boldsymbol{O}$, for a drawn copolymer.

larization $P_{\mathrm{r}}$ in an undrawn $80 / 20$ copolymer. The Young's modulus gradually increases with increasing remanent polarization $P_{\mathrm{r}}$. Although the piezoelectric constant $d_{31}$ initially increases with increasing $P_{\mathrm{r}}$, it tends to saturate at high $P_{\text {r. }}$.

The uniaxial drawing resulted in a strong inplane anisotropy of the piezoelectric constant, as shown in Figure 3. The value of $d_{31}$ is much larger than that of $d_{32}$ at a given $P_{\mathrm{r}}$. Both $d_{31}$ and $d_{32}$ are linearly proportional to $P_{\mathrm{r}}$.

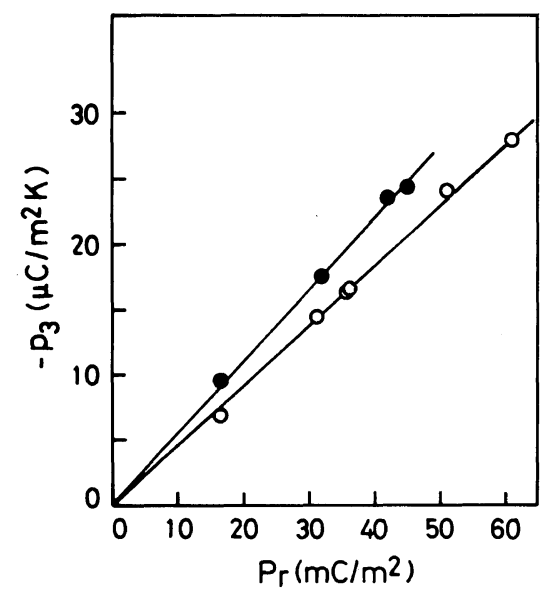

Figure 6. Dependence of the pyroelectric constant $p_{3}$ on $P_{\mathrm{r}}$ in undrawn and uniaxially drawn $\mathrm{VDF} / \mathrm{TeFE}$ copolymers. $\bigcirc$, for an undrawn copolymer; $\boldsymbol{O}$, for a drawn copolymer.

Young's moduli $1 / s_{11}$ and $1 / s_{22}$ are identical to each other and independent of $P_{\mathrm{r}}$. Similar behavior has been reported for uniaxially drawn and poled PVDF. ${ }^{20}$

Figure 4 shows the dependence of piezoelectric constants $e_{31}^{\mathrm{a}}$ and $e_{32}^{\mathrm{a}}$ on $P_{\mathrm{r}}$ for undrawn and uniaxially drawn $\mathrm{VDF} / \mathrm{TeFE}$ copolymers. Both $e_{31}^{\mathrm{a}}$ and $e_{32}^{\mathrm{a}}$ are linearly proportional to $P_{\mathrm{r}}$. The ratio of $e_{31}^{\mathrm{a}} / P_{\mathrm{r}}$ for the undrawn sample is about 0.42 , which is very close to the value reported for undrawn VDF/ TrFE copolymers. ${ }^{19}$ The ratios of $e_{31}^{\mathrm{a}} / P_{\mathrm{r}}$ and $e_{32}^{\mathrm{a}} / P_{\mathrm{r}}$ for uniaxially drawn samples are 0.9 and 0.2 respectively. These values are similar to those in drawn PVDF and VDF rich copolymers with TrFE. The results indicate a similarity in the mechanism of piezoelectric activity of these polymers.

Figure 5 shows the dependence of the longitudinal piezoelectric constant $d_{33}^{\mathrm{a}}$ on $P_{\mathrm{r}}$ for undrawn and uniaxially drawn samples. The values of $d_{33}^{\mathrm{a}}$ are linearly proportional to the remanent polarization $P_{\mathrm{r}}$. At a given value of $P_{\mathrm{r}}$, uniaxially drawn samples have larger values of $d_{33}^{\mathrm{a}}$ than undrawn samples.

The pyroelectric constants of undrawn and drawn copolymers were determined as a func- 


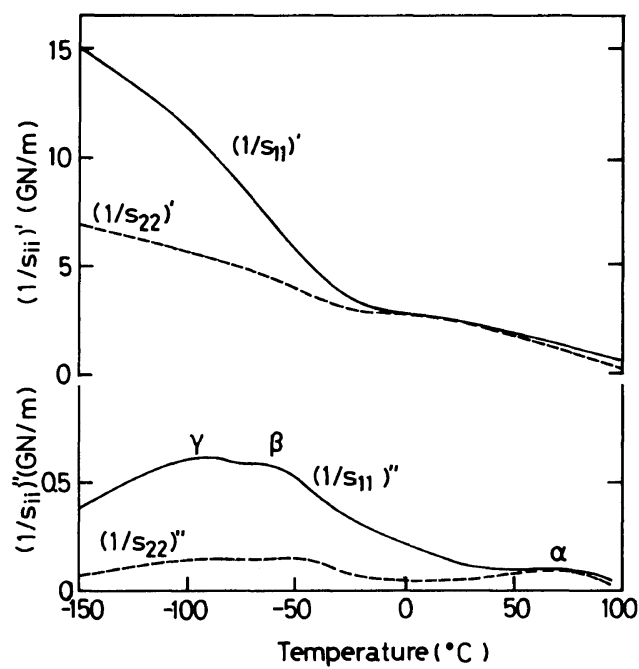

Figure 7. Temperature dependence of real and imaginary parts of Young's moduli of drawn VDF/TeFE copolymer.

tion of $P_{\mathrm{r}}$, and the results are shown in Figure 6. A linear relationship between $p_{3}$ and $P_{\mathrm{r}}$ is observed. The drawn samples have slightly larger pyroelectric activity than undrawn samples.

Figure 7 shows the temperature dependence of the real and imaginary parts of Young's moduli $1 / s_{11}$ and $1 / s_{22}$ of drawn VDF/TeFE copolymer. Three relaxations are observed, and designated as $\alpha, \beta$, and $\gamma$ in order of decreasing temperature. The $\alpha$ process near $60^{\circ} \mathrm{C}$ is attributable to the molecular motions in the crystalline regions. The $\beta$ process reflects micro-Brownian motions of the molecular chain backbone in the noncrystalline regions. ${ }^{21}$ The $\gamma$ process is related to local motions of the molecular chains. At low temperatures, the Young's modulus along the drawn direction is much larger than that perpendicular to the drawn direction.

Figure 8 shows the temperature dependence of the dielectric constant measured at $10 \mathrm{~Hz}$ for the drawn copolymer. Two relaxation processes are observed near 60 and $-40^{\circ} \mathrm{C}$ corresponding to the $\alpha$ and $\beta$ processes, respectively. The $\gamma$ process is not clearly ob-

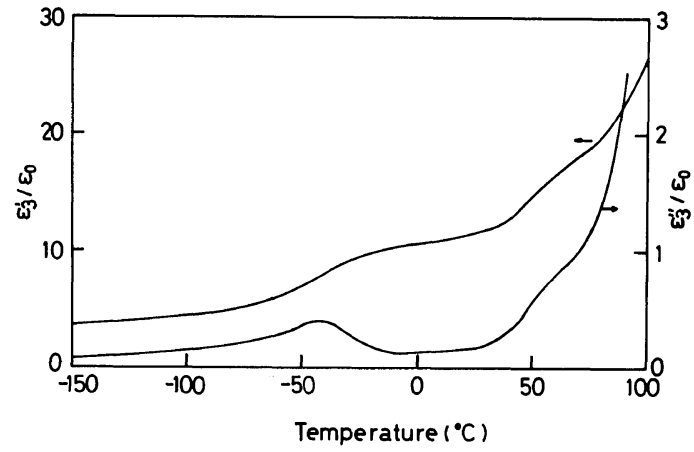

Figure 8. Temperature dependence of the dielectric constant of drawn VDF/TeFE copolymer.

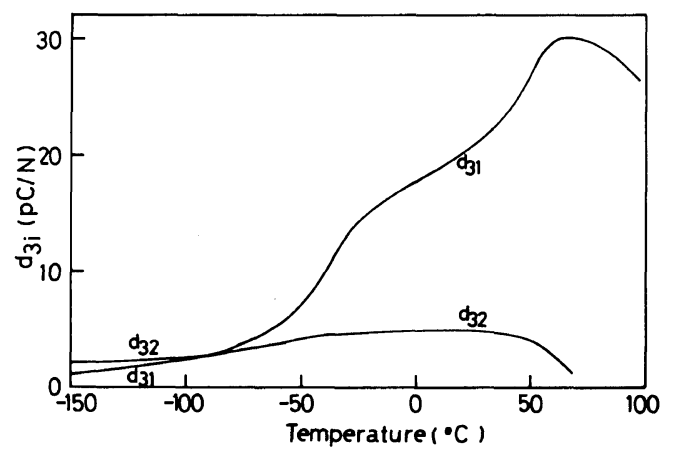

Figure 9. Temperature dependence of the $d_{31}$ and $d_{32}$ in an uniaxially drawn VDF/TeFE copolymer.

served.

Figure 9 shows the temperature dependence of the piezoelectric constants, $d_{31}$ and $d_{32}$, measured at $10 \mathrm{~Hz}$ for the drawn copolymer having a remanent polarization $P_{\mathrm{r}}=53$ $\mathrm{mC} \mathrm{m}^{-2}$. The value of $d_{31}$ is very small at low temperatures and increases rapidly around the glassy transition temperature $T_{\mathrm{g}}$ of $c a .-40^{\circ} \mathrm{C}$. Above $60^{\circ} \mathrm{C}, d_{31}$ gradually decreases with increasing temperature because of depolarization. After heating to $100^{\circ} \mathrm{C}$, the remaining $d_{31}$ was about $1 / 3$ of the original value. Such an anisotropy of $d_{31}$ and $d_{32}$ is very similar to that of PVDF. ${ }^{22}$

Below $-90^{\circ} \mathrm{C}, d_{32}$ is larger than $d_{31}$. However, $d_{32}$ shows only a small increase with temperature. At a room temperature, $d_{32}$ is much smaller than $d_{31}$. The sharp decrease in $d_{32}$ above $60^{\circ} \mathrm{C}$ also reflects depolarization of 


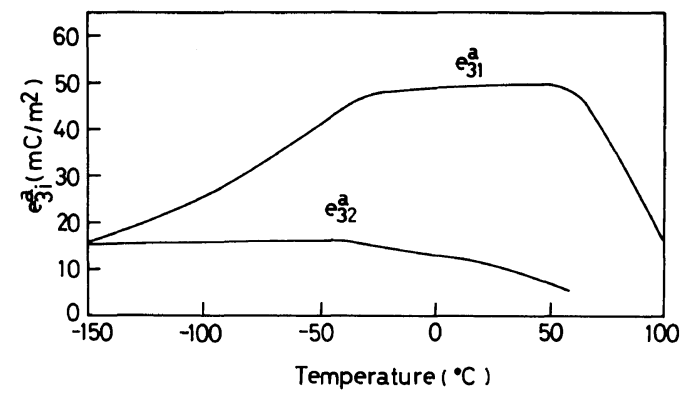

Figure 10. Temperature dependence of $e_{31}^{\mathrm{a}}$ and $e_{32}^{\mathrm{a}}$ in an uniaxially drawn VDF/TeFE copolymer.

crystalline regions.

Figure 10 shows the temperature dependence of piezoelectric constants $e_{31}^{\mathrm{a}}$ and $e_{32}^{\mathrm{a}}$ for the same sample. The $e_{31}^{\mathrm{a}}$ value shows a gradual increase up to $-30^{\circ} \mathrm{C}$ and then levels off. Above $60^{\circ} \mathrm{C}, e_{31}^{\mathrm{a}}$ suddenly decreases because of depolarization. The temperature dependence of $e_{31}^{\mathrm{a}}$ for the drawn VDF/TeFE copolymer is very similar to that of $\beta$-PVDF. ${ }^{23}$ The depolarization occurs more rapidly in the copolymer than in PVDF because the former has a lower melting point $\left(\sim 130^{\circ} \mathrm{C}\right)$ than the latter $\left(\sim 170^{\circ} \mathrm{C}\right)$.

$e_{32}^{\mathrm{a}}$ has the same value as $e_{31}^{\mathrm{a}}$ at $-150^{\circ} \mathrm{C}$ and rather decreases with increasing temperature around room temperature (above $T_{\mathrm{g}}$ ).

\section{DISCUSSION}

Various mechanisms have been proposed to explain the piezoelectric activity in PVDF. Broadhurst and coworkers have considered a model consisting of polarizable and liberating dipoles. ${ }^{17}$ Wada and Hayakawa developed a theory on piezo- and pyroelectricity for a semicrystalline polymer with polar crystals. ${ }^{24}$ Although their theoretical consideration is based on different models, they have derived a common mechanism called the dimensional effect which assumes the consistent dipoles to be rigid when a sample is subjected to deformation. The expected change in induced charges on deformation may be proportional to a change in thickness. Therefore, the $e$ constants are given as follows:

$$
\begin{aligned}
& e_{31}=e_{32}=0 . \\
& e_{33}=-P_{\mathrm{r}}
\end{aligned}
$$

We usually carry out direct measurements on the $d$ constant rather than on the true $e$ constant. The apparent $e^{\mathrm{a}}$ constant is easily obtained from the $d$ constant via. eq 8 . The dimensional effect leads to the expression for $e^{\mathrm{a}}$ as follows:

$$
\begin{aligned}
& e_{31}^{\mathrm{a}}=P_{\mathrm{r}} v_{31}, \\
& e_{32}^{\mathrm{a}}=P_{\mathrm{r}} v_{32}, \\
& e_{33}^{\mathrm{a}}=-P_{\mathrm{r}} .
\end{aligned}
$$

These expressions are independent of the choice of model. If the piezoelectric constants are measured as a function of macroscopic polarization $P_{\mathrm{r}}$, the contribution of the dimensional effect is estimated by eq $9-13$.

We previously investigated the piezoelectricity and pyroelectricity in VDF/TrFE copolymers in detail, ${ }^{19}$ and found the piezoelectric activity in undrawn copolymers primarily to arise from macroscopic dimensional change, indicating the molecular dipoles to be relatively rigid. The additional contribution from the electrostrictive coupling has been shown important in drawn copolymers of equimolar composition.

We measured various piezoelectric constants in the $80 / 20 \mathrm{VDF} / \mathrm{TeFE}$ copolymer as a function of $P_{\mathrm{r}}$. In undrawn samples, we obtained the ratio of $e_{31}^{\mathrm{a}} / P_{\mathrm{r}}$ of 0.42 . This value may be close to Poisson's ratio in usual semicrystalline polymers. Thus, the piezoelectric activity in undrawn VDF/TrFE is primarily attributable to the dimensional effect.

To evaluate true piezoelectric constants $e_{31}$ and $e_{33}$, we used the following equations ${ }^{19}$

$$
e_{33}=\frac{1-v}{(1-2 v)(1+v)} \frac{d_{33}^{\mathrm{a}}}{s}
$$




$$
e_{31}=\frac{1}{1-v} \cdot e_{31}^{\mathrm{a}}+\frac{v(1-v)}{(1-2 v)(1+v)} \frac{d_{33}^{\mathrm{a}}}{s}
$$

where $s$ is the elastic compliance and $v$, the Poisson's ratio; these are two independent quantities characterizing an isotropic elastic body. For the case of $P_{\mathrm{r}}=50 \mathrm{mC} \mathrm{m}^{-2}$, we have $d_{33}^{\mathrm{a}}=-11 \mathrm{pC} \mathrm{N}^{-1}$ from the results in Figure 5 and $1 / s=2.8 \mathrm{GN} \mathrm{m}^{-2}$ from Figure 2. Assuming that $v$ is 0.38 , we obtain $e_{33}$ as -58 $\mathrm{mC} \mathrm{m}^{-2}$, which is only $20 \%$ greater than $P_{\mathrm{r}}$. Thus, the value of $d_{33}^{\mathrm{a}}$ is also consistent with the prediction of the dimensional effect.

The drawing of the copolymer results in a piezoelectric anisotropy indicating $d_{31}$ to be much larger than $d_{32}$. Because Young's moduli $1 / s_{11}$ and $1 / s_{22}$ are identical, there is the same anisotropy, $e_{31}^{\mathrm{a}} \gg e_{32}^{\mathrm{a}}$. From Figure 4, we have the ratios, $e_{31}^{\mathrm{a}} / P_{\mathrm{r}}=0.9$ and $e_{32}^{\mathrm{a}} / P_{\mathrm{r}}=0.2$. If the dimensional effect is dominant, these ratios should be comparable to corresponding Poisson's ratios. It has been shown that $v_{31}$ of drawn semicrystalline polymers can exceed 0.5 , e.g., Tasaka and Miyata reported a value of 0.7 for $v_{31}$ of PVDF. ${ }^{25}$ Thus the value of 0.9 for $e_{31}^{\mathrm{a}} / P_{\mathrm{r}}$ in the VDF/TeFE copolymer may be broadly consistent with the prediction from the dimensional effect. Although $v_{32}$ for the $\mathrm{VDF} / \mathrm{TeFE}$ copolymer is not available so far, the value of 0.2 for $e_{32}^{\mathrm{a}} / P_{\mathrm{r}}$ may also be consistent with the corresponding Poisson's ratio. $^{26}$

Stefanou measured the longitudinal piezoelectric constants $d_{33}$ for VDF/TeFE copolymers of composition ranging from 61/39 to $96 / 4$ molar ratio. ${ }^{15} \mathrm{He}$ used an experimental set-up similar to ours, where a sample was sandwiched between a pair of brass electrodes. In such a configuration, we consider the inplane deformation of a sample to be restricted and hence, the piezoelectric constant obtained to be $d_{33}^{\mathrm{a}}$ rather than the true $d_{33}$. He reported a value of $-6.2 \mathrm{pC} \mathrm{N}^{-1}$ for $d_{33}$ in his notation with respect to the $78 / 22$ copolymer. This value corresponds to $d_{33}^{\mathrm{a}}$ of our sample with $P_{\mathrm{r}}=20 \mathrm{mC} \mathrm{m}^{-2}$.
In Figures 9 and 10, we show the temperature dependence of the piezoelectric constants. Both $d_{31}$ and $d_{32}$ increase with temperature except above $60^{\circ} \mathrm{C}$ where the disordering of aligned dipoles takes place. The temperature dependence of $e_{31}^{\mathrm{a}}$ and $e_{32}^{\mathrm{a}}$ is less than that of the $d$ constant. If the dimensional effect is dominant, the curves in Figure 10 should reflect a temperature dependence of $e_{31}^{\mathrm{a}}$ in the $\mathrm{VDF} / \mathrm{TeFE}$ copolymer similar to that in PVDF. $^{20}$

Sussner ${ }^{27}$ has discussed the temperature dependence of Poisson's ratios using Ohigashi's data for the temperature dependence of the electromechanical coupling factor $k_{31}$ and $k_{33}{ }^{28}$ Going a simple assumption that only the longitudinal piezoelectric constant $e_{33}$ is essential and independent of temperature while $e_{31}$ and $e_{32}$ are zero, Sussner derived the change in Poisson's ratio $v_{31}$ with temperature, that is, $v_{31}$ increases from $0.2\left(-100^{\circ} \mathrm{C}\right)$ to $0.6\left(50^{\circ} \mathrm{C}\right)$. His assumption is equivalent to the prediction from the dimensional effect in terms of the anisotropy of the true $e$ constant. We found in the VDF/TeFE copolymer that $e_{31}^{\mathrm{a}}$ at a room temperature is three times that at $-150^{\circ} \mathrm{C}$. As shown in Figure 10 , this can be interpreted in terms of an increase in $v_{31}$ by a factor of three which is same as the prediction by Sussner in PVDF.

The $e_{32}^{\text {a }}$ value remains constant up to $-40^{\circ} \mathrm{C}$ and then gradually decreases with increasing temperature. Such behavior may reflect the temperature dependence of $v_{32}$.

From these considerations, we can conclude that the piezoelectric activity in an $80 / 20$ copolymer of $\mathrm{VDF} / \mathrm{TeFE}$ primarily arises from macroscopic dimensional changes in a similar manner as in PVDF and VDF-rich copolymers with TrFE. However, the observed ratio of $e_{31}^{\mathrm{a}} / P_{\mathrm{r}}$ is too large and that of $e_{32}^{\mathrm{a}}$, slightly small compared to the corresponding Poisson's ratio. There must be certain other effects, although they are minor. The electrostrictive coupling proposed in VDF/TrFE copolymers may be one factor responsible for the remain- 
ing contributions.

The pyroelectric constant $p_{3}$ for a mechanically free sample generally consists of two terms: ${ }^{18}$

$$
p_{3}=\left(\frac{\partial P_{3}}{\partial T}\right)_{x_{i}=0}+\sum_{i=1}^{3} e_{3 i} \alpha_{i}
$$

where $T$ is the temperature and $\alpha_{i}$, the thermal expansion coefficient. The first term, called the primary effect, expresses the intrinsic pyroelectricity which appears in a clamped sample. The second term indicates coupling of the piezoelectricity and thermal expansion, and is called the secondary effect. We can calculate the secondary effect using true $e_{31}$ and $e_{33}$, if the thermal expansion coefficient $\alpha_{i}$ is known. Since the dimensional effect is dominant in the piezoelectricity of the VDF/TeFE copolymer, $e_{31}$ and $e_{32}$ are negligible and $e_{33}$ is nearly equal to $-P_{\mathrm{r}}$. From Figure 6 , we obtain the values of $-4.7 \times 10^{-4} \mathrm{~K}^{-1}$ and $-5.4 \times 10^{-4}$ $\mathrm{K}^{-1}$ for $p_{3} / P_{\mathrm{r}}$ in undrawn and drawn copolymers, respectively. These values were compared to the thermal expansion coefficient $\alpha_{3}$ in the thickness direction in order to evaluate the contribution from the secondary effect. Koizumi and coworkers reported the value of $1.10 \times 10^{-4} \mathrm{~K}^{-1}$ for the thermal expansion coefficient of $\mathrm{VDF} / \mathrm{TeFE}$ copolymer of composition $79 / 21 .^{21}$ We can take $1.10 \times 10^{-4} \mathrm{~K}^{-1}$ as the thermal expansion of the copolymer. The result indicates that the secondary effect contributes only $1 / 4$ the total pyroelectric activity in the undrawn $80 / 20 \mathrm{VDF} / \mathrm{TeFE}$ copolymer. The remaining is attributed to the primary effect which may be due to the reversible disordering of the dipoles.

\section{CONCLUSIONS}

The $d_{31}, d_{32}, d_{33}^{\mathrm{a}}$ and pyroelectric constant $p_{3}$ were measured for undrawn and uniaxially drawn VDF/TeFE copolymers of molar composition $80 / 20$ as a function of the remanent polarization $P_{\mathrm{r}}$. The ratio of the activity to $P_{\mathrm{r}}$ indicates that the dimensional effect is the primary contribution to piezoelectric activity in this copolymer. The secondary pyroelectric effect contributes $1 / 4$ the total activity.

Acknowledgement. The author would like to thank Prof. N. Koizumi of Kyoto University and Dr. T. Furukawa of the Institute of Physical and Chemical Research for their useful suggestions and discussion during this work. He wishes to thank Dr. E. Fukada, and Dr. H. Sasabe, and Dr. M. Date of the Institute of Physical and Chemical Research for their useful comments. $\mathrm{He}$ is also grateful to Mr. J. Sako of Daikin Kogyo Co., Ltd., for providing copolymer samples.

\section{REFERENCES}

1. H. Kawai, Jpn. J. Appl. Phys., 8, 976 (1969).

2. J. G. Bergman, Jr., J. H. Mcfee, and G. R. Grane, Appl. Phys. Lett., 18, 203 (1971).

3. R. Hayakawa and Y. Wada, Adv. Polym. Sci., 11, 1 (1973).

4. N. Murayama, K. Nakamura, H. Obara, and M. Segawa, Ultrasonics, 14, 15 (1976).

5. R. G. Kepler, Ann. Rev. Phys., Chem. 29, (1979).

6. E. Fukuda, Proceedings of the 2nd Meeting on Ferroelectric Materials and Their Applications, Kyoto, 1979.

7. R. G. Kepler and R. A. Anderson, J. Appl. Phys., 49, 1232 (1978).

8. D. Naegele and D. Y. Yoon, Appl. Phys. Lett., 33, 132 (1978).

9. T. Furukawa, M. Date, and E. Fukada, J. Appl. Phys., 51, 1135 (1980).

10. T. Furukawa and G. E. Johnson, Appl. Phys. Lett., 38, 1027 (1981).

11. J. B. Lando and W. W. Doll, J. Macromol. Sci. Phys., B, 2, 205 (1968).

12. A. I. Baise, H. Lee, B. Oh, R. E. Salomon, and M. M. Labes, Appl. Phys. Lett., 26, 428 (1975).

13. G. T. Davis and M. G. Broadhurst, "Piezoelectricity and Pyroelectricity in polar polymers," in International Symposium on Electrets and Dielectrics," M. S. de Campos, Ed., Academia Brasileira de Ciencias, Rio de Janeiro, 1977, p 299.

14. R. E. Collins, M. G. Broadhurst, and G. T. Davis, "Studies in Electrical and Electronic Engineering, 2, Charge storage, Charge Transport and Electrostatics with their Applications," Y. Wada, M. M. Perlman, and H. Kokado Ed., 1979.

15. H. Stefanou, J. Appl. Phys., 50, 1480 (1979).

16. J. C. Hicks, T. E. Jones, and J. C. Logan, J. Appl. 
Phys., 51, 1135 (1980).

17. M. G. Broadhurst, G. T. Davis, J. E. McKinney, and R. E. Collions, J. Appl. Phys., 49, 4992 (1978).

18. W. G. Cady, "Piezoelectricity," Dover, New York, 1964.

19. T. Furukawa, J. X. Wen, K. Suzuki, Y. Takashina, and M. Date, J. Appl. Phys., 56, 829 (1984).

20. T. Furukawa, J. Aiba, and E. Fukada, J. Appl. Phys., 50, 3615 (1979).

21. N. Koizumi, J. Hagino, and Y. Murata, Ferroelectrics, 32, 141 (1981).
22. E. Fukada and S. Takashita, Jpn. J. Appl. Phys., 8, 960 (1969).

23. M. Date, T. Furukawa, and E. Fukada, J. Appl. Phys., 51, 3830 (1980).

24. Y. Wada and R. Hayakawa, Ferroelectrics, 32, 116 (1981).

25. S. Tasaka and S. Miyata, Ferroelectrics, 32, 17 (1981).

26. J. X. Wen, Jpn. J. Appl. Phys., submitted.

27. H. Sussner, Phys. Lett., 58A, 426 (1976).

28. H. Ohigashi, J. Appl. Phys., 47, 949 (1976). 